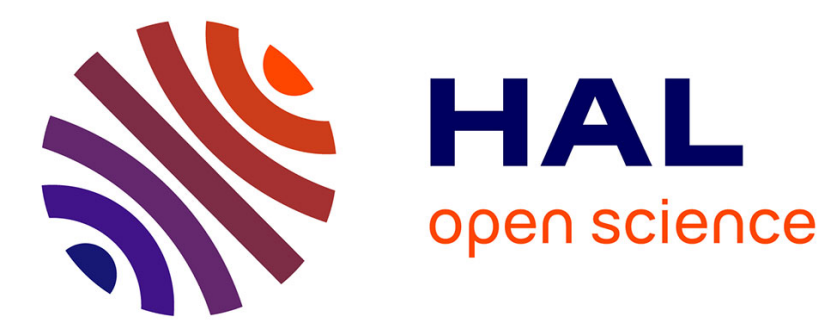

\title{
Variational principles for systems with unilateral constraints
}

V V Rumyantsev

\section{To cite this version:}

V V Rumyantsev. Variational principles for systems with unilateral constraints. Journal of Applied Mathematics and Mechanics, 2006, 70, pp.808 - 818. 10.1016/j.jappmathmech.2007.01.002 . hal01393694

\section{HAL Id: hal-01393694 \\ https://hal.science/hal-01393694}

Submitted on 8 Nov 2016

HAL is a multi-disciplinary open access archive for the deposit and dissemination of scientific research documents, whether they are published or not. The documents may come from teaching and research institutions in France or abroad, or from public or private research centers.
L'archive ouverte pluridisciplinaire HAL, est destinée au dépôt et à la diffusion de documents scientifiques de niveau recherche, publiés ou non, émanant des établissements d'enseignement et de recherche français ou étrangers, des laboratoires publics ou privés. 


\title{
Variational principles for systems with unilateral constraints
}

\author{
V.V. Rumyantsev \\ Moscow, Russia \\ E-mail address: vvrum@ccas.ru.
}

Derivations and formulations are given of the variational principles of analytical mechanics for systems with unilateral ideal smooth constraints, originally established for systems with bilateral constraints. The virtual work principle, the Fourier inequality, the d'Alembert-Lagrange principle, the Gauss principle of least constraint and its modification - the Chetayev principle of maximum work, the Jourdain principle, the Hamilton-Ostrogradskii principle, the principle of least action in Lagrangian and Jacobian forms, and the Suslov-Voronets principle are described.

\section{Some definitions $\mathbf{s}^{1,2}$}

Let $O x y z$ be an inertial system of coordinates relative to which a system of point masses $M_{v}$ with masses $m_{v}(\nu=1$, $\ldots, N)$ is moving. If the points $M_{\nu}$ of the system at an arbitrary instant of time $t$ are unable to occupy an arbitrary position in space, or are unable to have arbitrary velocities, then such a system is said to be constrained. The conditions imposed on the positions or motions of a constrained system are called constraints. If the constraints do not allow the system to occupy arbitrary positions, they are called geometric or finite constraints. If the constraints only do not allow the points of the system at a given instant of time to have arbitrary velocities, the constraints are called kinematic or differential constraints. Finite constraints, and also differential integrable constraints, are called holonomic constraints, while differential non-integrable constraints are called non-holonomic constraints. If the constraints do not depend on time explicitly, they are called stationary or scleronomic constraints, and if they do, they are called non-stationary or rheonomic constraints.

Constraints are said to be bilateral if analytically they are expressed by equations of the form

$$
f_{\alpha}\left(t, x_{v}, y_{v}, z_{v}\right)=0, \quad \varphi_{\beta}\left(t, x_{v}, y_{v}, z_{v}, \dot{x}_{v}, \dot{y}_{v}, \dot{z}_{v}\right)=0 ; \quad \alpha=1, \ldots, a ; \quad \beta=1, \ldots, b
$$

and unilateral if they are expressed by inequalities of the form

$$
f_{\alpha}\left(t, x_{v}, y_{v}, z_{v}\right) \geq 0, \quad \varphi_{\beta}\left(t, x_{v}, y_{v}, z_{v}, \dot{x}_{v}, \dot{y}_{v}, \dot{z}_{v}\right) \geq 0
$$

When the left-hand sides of these relations are positive, the constraints are said to be loosened, or that the system has left its constraints; when, however, the left-hand sides are equal to zero, the constraints are active, or strained. Constraints (1.1) and (1.2) will be assumed to be independent and smooth. We will confine ourselves below to linear 
differential constraints

$$
\varphi_{\beta}\left(t, x_{v}, y_{v}, z_{\mathrm{v}}, \dot{x}_{v}, \dot{y}_{v}, \dot{z}_{\mathrm{v}}\right)=\sum_{v} \mathbf{B}_{v}^{\beta} \cdot \dot{\mathbf{r}}_{\mathrm{v}}+D_{\beta} \geq 0 ; \quad \mathbf{r}_{\mathrm{v}}=\left(x_{\mathrm{v}}, y_{\mathrm{v}}, z_{\mathrm{v}}\right), \dot{\mathbf{r}}_{\mathrm{v}}=\frac{d \mathbf{r}_{\mathrm{v}}}{d t}
$$

where $\mathbf{B}_{v}^{\beta}$ and $D_{\beta}$ are certain functions of time and the coordinates.

Here and below, summing over $v$ is carried out from $\nu=1$ to $\nu=N$, summing over $\alpha$ is carried out from $\alpha=1$ to $\alpha=a$ and summing over $\beta$ is carried out from $\beta=1$ to $\beta=b$.

Elementary displacements $\delta \mathbf{r}_{\nu}$, satisfying the conditions

$$
\delta f_{\alpha}=\sum_{v} \operatorname{grad}_{r_{v}} f_{\alpha} \cdot \dot{\mathbf{r}}_{v} \geq 0, \quad \sum_{v} \operatorname{grad}_{\dot{\mathbf{r}}_{v}} \varphi_{\beta} \cdot \delta \mathbf{r}_{v} \geq 0
$$

at a fixed value of time $(\delta t=0)$, are called virtual displacements of points of the system, and here the equality sign only occurs for non-loosening displacements which leave the system constrained.

Note that here there is a considerable defference between the virtual displacements in the cases of bilateral and unilateral constraints. In the former case, the virtual displacements are always reversible, whereas in the latter case they are irreversible, with the exception only of virtual displacements which leave the system constrained; this explains the double sign - equal to or greater than zero. Figuratively speaking, in the case of bilateral constraints, the system lies somewhere within configuration space, and its virtual displacements can occur in any direction submitted by the constraints, whereas in the case of unilateral constraints, the system lies on the boundary of the configuration space, as a result of which, when the virtual displacements are directed inwards, displacements in the opposite direction are impossible as they would lead beyond the configuration space. ${ }^{3}$

For the motion of a mechanical system under the action of prescribed forces $\mathbf{F}_{v}$ applied, the constraints imposed on the system act at different points $M_{\nu}$ of the system with certain forces $\mathbf{R}_{v}$ called constraint forces.

\subsection{Axiom of constraints}

The action of constraints imposed on a mechanical system can be replaced by the action of constraint forces $\mathbf{R}_{v}$.

\subsection{Corollary}

If to the prescribed forces $\mathbf{F}_{v}$ acting on points of the system, constraint forces $\mathbf{R}_{v}$ are added, then the system can be thought of as being free of the constraints causing the constraint forces $\mathbf{R}_{v}$.

The constraints imposed on the system depend on the physical nature of the mechanisms implementing the constraints, and therefore a constraint characteristic should be introduced into the mechanics in the form of an axiom that fixes realistic relations observed in experiments: In many cases the concept of ideal constraints is adopted as such an axiom. By definition, the total elementary work of ideal constraint forces $\mathbf{R}_{v}$ on an arbitrary virtual displacement of the system $\delta \mathbf{r}$ is equal to zero

$$
\sum_{v} \mathbf{R}_{v} \cdot \delta \mathbf{r}_{v}=0
$$

in the case of bilateral constraints or equal to or greater than zero in the case of unilateral constraints

$$
\sum_{v} \mathbf{R}_{v} \cdot \delta \mathbf{r}_{v} \geq 0
$$

and here the equality sign occurs only for non-loosening displacements.

We will now find expressions for the constraint forces corresponding to ideal constraints. We will first consider the non-loosening virtual displacements $\delta \mathbf{r}_{v}$ for which, in relations (1.3), the equality signs apply. We will multiply each of Eq. (1.3) by arbitrary multipliers $-\lambda_{\alpha}$ and $-\mu_{\beta}$, sum them and then add them to Eq. (1.4); as a result, we obtain the equation

$$
\sum_{v}\left(\mathbf{R}_{v}-\sum_{\alpha} \lambda_{\alpha} \operatorname{grad}_{r_{v}} f_{\alpha}-\sum_{\beta} \mu_{\beta} \operatorname{grad}_{\mathbf{r}_{v}} \varphi_{\beta}\right) \cdot \delta \mathbf{r}_{v}=0
$$


from which we find expressions for the ideal constraint forces

$$
\mathbf{R}_{v}=\sum_{\alpha} \lambda_{\alpha} \operatorname{grad}_{r_{v}} f_{\alpha}+\sum_{\beta} \mu_{\beta} \operatorname{grad}_{\mathbf{r}_{v}} \varphi_{\beta}, \quad v=1, \ldots, N
$$

where the number of constraint multipliers $\lambda_{\alpha}$ and $\mu_{\beta}$ is equal to the number $a+b$ of constraint equations (1.1). In the sums on the right-hand side of Eq. (1.5), each term represents the action of one respective constraint on a given point $M_{\nu}$

$$
\mathbf{R}_{v}^{\alpha}=\lambda_{\alpha} \operatorname{grad}_{r_{v}} f_{\alpha}, \quad \mathbf{R}_{v}^{\beta}=\mu_{\beta} \operatorname{grad}_{\dot{r}_{v}} \varphi_{\beta}
$$

In the case of unilateral constraints, we will retain expressions (1.6) for the constraint forces, on account of which the expression for the virtual work of the unilateral constraint forces coincides in form with the respective expression for bilateral constraints, i.e.,

$$
\sum_{v} \mathbf{R}_{v} \cdot \delta \mathbf{r}_{v}=\sum_{\alpha} \lambda_{\alpha} \operatorname{grad}_{r_{v}} f_{\alpha} \cdot \delta \mathbf{r}_{v}+\sum_{\beta} \mu_{\beta} \operatorname{grad}_{\mathbf{r}_{v}} \varphi_{\beta} \cdot \delta \mathbf{r}_{v}
$$

whence, by virtue of relation (1.4), there follow additional conditions for the multipliers

$$
\lambda_{\alpha} \geq 0, \quad \mu_{\beta} \geq 0 ; \quad \alpha=1, \ldots, a ; \quad \beta=1, \ldots, b
$$

It should be pointed out that, in the case of loosening virtual displacements, the left-hand side of relation (1.4) represents the elementary work of the constraint forces only in the nominal sense, namely if it is assumed that, during the displacement, the constraint forces retain their initial values. In this sense, inequality (1.4) can be regarded as indicating the relationship between the directions of the displacements and the constraint forces, rather than the work of the constraint forces. The work of an ideal unilateral constraint force on virtual displacement is always equal to zero: when the displacement leaves the system constrained, then

$$
\sum_{v} \operatorname{grad}_{r_{v}} f_{\alpha} \cdot \delta \mathbf{r}_{v}=0, \quad \operatorname{grad}_{\mathbf{r}_{v}} \varphi_{\beta} \cdot \delta \mathbf{r}_{v}=0
$$

but when the displacement causes system constraint violation, i.e. when

$$
\sum_{v} \operatorname{grad}_{r_{v}} f_{\alpha} \cdot \delta \mathbf{r}_{v}>0, \quad \operatorname{grad}_{\mathbf{r}_{v}} \varphi_{\beta} \cdot \delta \mathbf{r}_{v}>0
$$

the corresponding reactions vanish, and consequently $\lambda_{\alpha}=0$ and $\mu_{\beta}=0(\alpha=1, \ldots, a ; \beta=1, \ldots, b) .{ }^{1}$

In the literature, non-holonomic unilateral contraints are considered in two versions. In the first of these, the conditions for the coordinates and velocities are written in the form of inequalities, as in the present paper. In the second version, inequalities link the constraint forces, and the coordinates and velocities satisfy the constraint equations as long as the constraint forces belong to some open set in constraint force space. As soon as the constraint force reach the boundary of this set, the constraints disappear. This version is not considered in the present paper.

\section{The virtual work principle, the fourier inequality}

The equilibrium position of a system under the action of given forces is that position of the system in which it may for an indefinite time be at rest relative to a given reference system. The equilibrium positions of systems are usually considered when the prescribed forces $\mathbf{F}_{v}$ and constraints do not depend on time explicitly.

Let us complete the prescribed forces $\mathbf{F}_{v}$ with all constraint forces $\mathbf{R}_{v}$; then the mechanical system, according to the axiom of constraints, can be thought of as a system of free points under the action of forces $\mathbf{F}_{v}+\mathbf{R}_{v}$. For free points, the equilibrium equations have the form ${ }^{4}$

$$
\mathbf{F}_{v}+\mathbf{R}_{v}=0, \quad v=1, \ldots, N
$$


We multiply these equations scalarly by the virtual displacements $\delta \mathbf{r}_{v}$ of points of the system, sum them and, taking into account inequality (1.4), obtain the relation

$$
\sum_{v} \mathbf{F}_{v} \cdot \delta \mathbf{r}_{v} \leq 0
$$

which holds for arbitrary virtual displacements $\delta \mathbf{r}_{v}$ from the equilibrium position. Relation (2.1) is not only necessary but also sufficient ${ }^{1,2}$ for equilibrium, i.e., it expresses the principle called the virtual work principle: for the equilibrium of the system the sum of elementary work of active forces on any virtual displacement of the system is either zero or negative; it is zero for non-loosening displacements and negative for loosening displacements.

This principle for the case of bilateral constraints was established by Bernoulli, and for the case of unilateral constraints by Fourier. ${ }^{5}$

In the case of potential forces, when

$$
\mathbf{F}_{v}=\operatorname{grad}_{r_{v}} U, \quad v=1, \ldots, N
$$

where $U\left(\mathbf{r}_{1}, \ldots, \mathbf{r}_{N}\right)$ is the force function, inequality (2.1) takes the form

$$
\delta U \leq 0
$$

\section{The d'Alembert-Lagrange principle}

Let us consider the motion of a system subject to ideal constraints (1.1) and (1.2). Taking into account expressions (1.5) for the forces of the strained constraints, we can represent the equations of motion in the form

$$
\mathbf{F}_{v}-m_{v} \ddot{\mathbf{r}}_{v}+\sum_{\alpha} \lambda_{\alpha} \operatorname{grad}_{r_{v}} f_{\alpha}+\sum_{\beta} \lambda_{\beta} \operatorname{grad}_{\mathbf{r}_{v}} \varphi_{\beta}=0, \quad v=1, \ldots, N
$$

Multiplying each of the equations in system (3.1) scalarly by $\delta \mathbf{r}_{\nu}$, and summing over all $v=1, \ldots, N$, we obtain

$$
\sum_{v}\left(\mathbf{F}_{v}-m_{v} \ddot{\mathbf{r}}_{v}\right) \cdot \delta \mathbf{r}_{v}+\sum_{\alpha} \lambda_{\alpha} \delta f_{\alpha}+\sum_{\beta} \mu_{\beta} \operatorname{grad}_{\mathbf{r}_{v}} \varphi_{\beta} \cdot \delta \mathbf{r}_{v}=0
$$

from which, taking into account inequality (1.4), we obtain an analytical expression for the d'Alembert-Lagrange principle

$$
\sum_{v}\left(\mathbf{F}_{v}-m_{v} \ddot{\mathbf{r}}_{v}\right) \cdot \delta \mathbf{r}_{v} \leq 0
$$

The sum of the elementary work of active forces $\mathbf{F}_{v}$ and inertia forces $-m_{\nu} \ddot{r}_{v}$ on any virtual displacement is equal to zero or is non-positive, depending on whether all constraints are bilateral or whether there are unilateral constraints among them; the equality sign occurs in the case of non-loosening virtual displacements, and the less than sign in the case of loosening displacements.

Principle (3.3) was derived as a consequence of the equations of motion (3.1). It is possible, conversely, to adopt principle (3.3) as the primary one and, from it, then to obtain the equations of motion (3.1).

Relation (3.3) defines the relationship between the virtual displacements $\delta \mathbf{r}_{v}$ that are allowed by constraints (1.1) and (1.2) imposed, the active forces $\mathbf{F}_{v}$ applied to the system and the accelerations $\ddot{r}_{v}$ caused by the prescribed forces under the constraints imposed.

When all the accelerations $\ddot{r}_{v}=0(\nu=1, \ldots, N)$ and, consequently, the system is in equilibrium, the d'Alembert-Lagrange principle (3.3) becomes the virtual work principle (2.1) (the Fourier inequality).

The quantity $P_{v}=F_{v}-m_{v} \ddot{r}_{v}$ is sometimes called the lost force. In terms of this quantity, the d'Alembert-Lagrange principle can be represented by an inequality similar to that of (2.1)

$$
\sum_{v} \mathbf{P}_{v} \cdot \delta \mathbf{r}_{v} \leq 0
$$


In the case of active potential forces, the d'Alembert-Lagrange principle takes the form

$$
\sum_{v}\left(\operatorname{grad}_{r_{v}} U-m_{v} \ddot{\mathbf{r}}_{v}\right) \cdot \delta \mathbf{r}_{v}=\delta U-\sum_{v} m_{v} \ddot{\mathbf{r}}_{v} \cdot \delta \mathbf{r}_{v} \leq 0
$$

where $U=U\left(t, \mathbf{r}_{1}, \ldots, \mathbf{r}_{N}\right)$.

Principle (3.3) was first obtained for bilateral constraints by Lagrange ${ }^{6}$ by combining the d'Alembert and Bernoulli principles, and for unilateral constraints by Ostrogradskii. ${ }^{7}$

\section{The Gauss principle of least constraint ${ }^{8}$}

In the d'Alembert-Lagrange principle, the simultaneous $(\delta t=0)$ positions $\mathbf{r}_{v}$ and $r_{v}^{\prime}$ of the system in the actual and any infinitely close kinematically possible motions, differing by quantities $\delta r_{v}=r_{v}^{\prime}-r_{v}$, satisfying conditions (1.3), are compared. Here, principle (3.3) does not have the nature of the principle of stationarity (extremality) of any function.

Gauss managed to convert the d'Alembert-Lagrange principle into the principle of least constraint by narrowing the set of motions compared: the actual motion is compared with kinematically possible motions in which, at a given instant of time $t$, the radius vectors $\mathbf{r}_{v}$ and velocities $\dot{\boldsymbol{r}}_{\nu}$ of the points $M_{\nu}$ of the system have the same values as in the actual motion. Gauss called these motions conceivable - for them, the accelerations of points $\dot{\boldsymbol{r}}_{\nu}$ that are possible with the prescribed configuration and velocity of the system are cosidered.

At the instant of time $t+\tau$, where $\tau$ is a small quantity, we have

$$
\mathbf{r}_{v}(t+\tau)=\mathbf{r}_{v}(t)+\dot{\mathbf{r}}_{v}(t) \tau+\frac{1}{2} \ddot{\mathbf{r}}_{\mathrm{v}}(t) \tau^{2}+\ldots
$$

Neglecting terms of the third and higher orders in $\tau$, with Gaussian variation, at the instant of time $t+d t$ we obtain

$$
\delta \mathbf{r}_{v}=\frac{1}{2}\left(\ddot{\mathbf{r}}_{v}^{\prime}-\ddot{\mathbf{r}}_{v}\right)(d t)^{2}=\frac{1}{2} \delta \ddot{\mathbf{r}}_{v}(d t)^{2}
$$

Substituting $\delta \mathbf{r}_{v}$ into expression (3.3) of the d'Alembert-Lagrange principle, we will have

$$
\sum_{v}\left(\mathbf{F}_{v}-m_{v} \ddot{\mathbf{r}}_{v}\right) \cdot \delta \ddot{\mathbf{r}}_{v} \leq 0
$$

or, taking into account that the prescribed active forces $\mathbf{F}_{v}=\mathbf{F}_{v}(t, \mathbf{r}, \dot{\mathbf{r}})$ are independent of the accelerations $\ddot{r}_{v}$, and introducing into cosideration the function

$$
Z=\frac{1}{2} \sum_{v} m_{v}\left(\ddot{\mathbf{r}}_{v}-\frac{\mathbf{F}_{v}}{m_{v}}\right)^{2}
$$

we will rewrite relation (4.1) in the form

$$
\delta Z=\sum_{v} m_{v}\left(\ddot{\mathbf{r}}_{v}-\frac{\mathbf{F}_{v}}{m_{v}}\right) \cdot \delta \ddot{\mathbf{r}}_{v} \geq 0
$$

from which $\delta^{2} Z=\sum_{v} m_{v}\left(\delta \ddot{r}_{v}\right)^{2}>0$.

Gauss called the quantity $Z$ [Eq. (4.2)] a constraint and adopted it as a measure of the deviation of the actual motion from the motion of a system free from all constraints. Consequently, for the actual motion, constraint $Z$ has the least value in the class of conceivable motions. This statement forms the Gauss principle of least constraint. For holonomic and linear non-holonomic constraints, the Gauss principle has the same generality as the d'Alembert-Lagrange principle. ${ }^{2}$

\section{Modification of Gauss principle: the Chetayev principle9}

Chetayev proposed an interesting modification of Gauss principle. Along with the actual motion of the system, we will examine the class of Gauss conceivable motions of the system $(\mu)$ in the time interval from $t$ to $t+d t$, determined at the instant of time $t$ by the values of $\mathbf{r}_{v}$ and $\dot{\boldsymbol{r}}_{v}=\mathbf{v}_{v}$, and by constraints (1.1) and (1.2) imposed on the system. 
The work of the prescribed forces $\mathbf{F}_{\nu}$ on an infinitesimal displacement

$$
\mathbf{r}_{v}(t+d t)-\mathbf{r}_{v}(t)=\left(\dot{\mathbf{r}}_{v}+\frac{1}{2} \delta \dot{\mathbf{r}}_{v}\right) d t
$$

of conceivable motion is equal to

$$
\sum_{v} \mathbf{F}_{v} \cdot\left(\dot{\mathbf{r}}_{v}+\frac{1}{2} \delta \dot{\mathbf{r}}_{\mathrm{v}}\right) d t
$$

where $\delta \dot{r}_{v}$ is the variation of velocity of a point $M_{v}$ within a time interval $d t$ for the conceivable motion. From this expression we will subtract the expression for the work on the examined displacement of the forces $m_{\nu} \ddot{r}_{v}$ that would be sufficient to create the actual motion if the points $M_{\nu}$ were completely free:

$$
\sum_{v} m_{v} \ddot{\mathbf{r}}_{\mathrm{v}} \cdot\left(\dot{\mathbf{r}}_{\mathrm{v}}+\frac{1}{2} \delta \dot{\mathbf{r}}_{\mathrm{v}}\right) d t
$$

As a result we obtain the expression

$$
A_{\mu}=\sum_{v}\left(\mathbf{F}_{v}-m_{v} \ddot{\mathbf{r}}_{v}\right) \cdot\left(\dot{\mathbf{r}}_{v}+\frac{1}{2} \delta \dot{\mathbf{r}}_{v}\right) d t
$$

for the work on an elementary cycle consisting of forward conceivable motion in a field of prescribed forces and backward (reverse) motion in a field of forces that would be sufficient to create the actual motion if the points of the system were completely free.

The work on a similar cycle constructed for the actual motion is equal to

$$
A=\sum_{v}\left(\mathbf{F}_{v}-m_{v} \ddot{\mathbf{r}}_{v}\right) \cdot\left(\dot{\mathbf{r}}_{v}+\frac{1}{2} d \dot{\mathbf{r}}_{v}\right) d t
$$

Subtracting Eq. (5.2) from Eq. (5.1), we obtain

$$
A_{\mu}-A=\sum_{v}\left(\mathbf{F}_{v}-m_{v} \ddot{\mathbf{r}}_{v}\right) \cdot\left(\delta \dot{\mathbf{r}}_{v}-d \dot{\mathbf{r}}_{v}\right) \frac{d t}{2}
$$

We will denote by $\Delta$ the operation of calculating the increment due to transition from the actual motion to a close conceivable motion. Since the forces $\mathbf{F}_{\nu}$ are independent of the accelerations, it follows that $\Delta \mathbf{F}_{\nu}=0$, and Eq. (5.3) can be represented in the form

$$
\Delta A=\frac{(d t)^{2}}{2} \sum_{v}\left(\mathbf{F}_{v}-m_{v} \ddot{\mathbf{r}}_{v}\right) \cdot \Delta \ddot{\mathbf{r}}_{v}=-\Delta \frac{(d t)^{2}}{2} \sum_{v} \frac{1}{2 m_{v}}\left(\mathbf{F}_{v}-m_{v} \ddot{\mathbf{r}}_{v}\right)^{2}
$$

According to the relation (4.1), we obtain

$$
\Delta A \leq 0
$$

Applying to this inequality the operation $\Delta$, we find

$$
\Delta^{2} A=-\frac{(d t)^{2}}{2} \sum_{v} m_{v}\left(\Delta \ddot{r}_{v}\right)^{2}<0
$$

Consequently, the Chetayev principle of maximum work holds: the work $A$ on an elementary cycle consisting of forward motion in the field of prescribed forces and reverse motion in the field of forces that would be sufficient to create the actual motion if the mechanical system were completely free has a maximum for the actual motion in the class of Gauss conceivable motions. 


\section{The Jourdain principle ${ }^{10}$}

Unlike the d'Alembert-Lagrange, Gauss and Chetayev principles, the actual motion in the Jourdain principle is compared with those kinematically possible motions for which at a given instant of time $t$ the radius vectors $\mathbf{r}_{v}$ of the points of the system are the same as in the actual motion.

With Jourdain variation, at the instant in time $t+d t$ we will have

$$
\delta \mathbf{r}_{v}=\left(\dot{\mathbf{r}}_{v}^{\prime}-\dot{\mathbf{r}}_{v}\right) d t=\delta \dot{\mathbf{r}}_{v} d t
$$

Substituting this expression into expression (3.3) of the d'Alembert-Lagrange principle, we obtain the Jourdain principle

$$
\sum_{v}\left(\mathbf{F}_{v}-m_{v} \ddot{\mathbf{r}}_{v}\right) \cdot \delta \dot{\mathbf{r}}_{v} d t \leq 0
$$

\section{The Hamilton-Ostrogradskii principle}

Let us consider the motion of a holonomic mechanical system in the time interval from $t_{0}$ to $t_{1}$, and also, for each instant $t$, the virtual displacements $\delta \mathbf{r}_{v}$ from position $\mathbf{r}_{v}$ occupied in the actual motion. We will that assume the virtual displacements $\delta \mathbf{r}_{v}$ are functions of $t$ that belong to class $C_{2}$ and vanish at instants of time $t_{0}$ and $t_{1}$ corresponding to possible positions $A_{0}$ and $A_{1}$ of the system in the configuration space. The sequence of positions $\mathbf{r}_{v}+\delta \mathbf{r}_{v}$ can be regarded as a varied or tortuous path that for a non-holonomic system does not, however, satisfy the constraint equations. ${ }^{1}$

The variation of the kinetic energy of the system is given by

$$
\delta T=\sum_{v} m_{v} \dot{\mathbf{r}}_{v} \cdot \delta \dot{\mathbf{r}}_{v}, \quad \text { with } \quad \delta \dot{\mathbf{r}}_{v}=\frac{d}{d t} \delta \mathbf{r}_{v}
$$

Integrating with respect to $t$, we will have

$$
\int_{t_{0}}^{t_{1}} \delta T d t=\int_{t_{0}}^{t_{1}} m \dot{\mathbf{r}}_{v} \cdot \frac{d \delta \mathbf{r}_{v}}{d t} d t=\left.\sum_{v} m_{v} \dot{\mathbf{r}}_{v} \cdot \delta \mathbf{r}_{v}\right|_{t_{0}} ^{t_{1}}-\int_{t_{0}}^{t_{1}} \sum_{v} m_{v} \ddot{\mathbf{r}_{v}} \cdot \delta \mathbf{r}_{v} d t
$$

The first term on the right-hand side of this equation is equal to zero, since, according to the condition, $\delta \mathbf{r}_{v}=0$ at instants of time $t_{0}$ and $t_{1}$, which implies

$$
\int_{t_{0}}^{t_{1}}\left(\delta T+\sum_{v} \mathbf{F}_{v} \cdot \delta \mathbf{r}_{v}\right) d t=-\int_{t_{0}}^{t_{1}} \sum_{v}\left(m_{v} \ddot{\mathbf{r}}_{v}-\mathbf{F}_{v}\right) \cdot \delta \mathbf{r}_{v} d t
$$

The right-hand side of this equation is non-positive according to the d'Alembert-Lagrange principle (3.3), so that

$$
\int_{t_{0}}^{t_{1}}\left(\delta T+\sum_{v} \mathbf{F}_{v} \cdot \delta \mathbf{r}_{v}\right) d t \leq 0 ; \quad \delta \mathbf{r}_{v}=0 \quad \text { with } t=t_{0}, t_{1}
$$

The Hamilton-Ostrogradskii principle states that the integral with respect to time of the sum of the variation of the kinetic energy and the work of the active forces on virtual displacements $\delta \mathbf{r}_{v}$ for the actual motion is equal to zero or is negative in comparison with motions along tortuous paths between the same positions $A_{0}$ and $A_{1}$ if the motion of the system along all paths is accomplished in the same time interval $t_{1}-t_{0}$, and here $t_{0}$ and $t_{1}$ are the initial and final instants of time that are common to all paths.

In the case of active potential forces, when $F_{v}=\operatorname{grad}_{r_{v}} U(\nu=1, \ldots, N)$, we obtain the Hamilton principle

$$
\int_{t_{0}}^{t_{1}} \delta L d t \leq 0 ; \quad \delta \mathbf{r}_{v}=0 \quad \text { with } t=t_{0}, t_{1}
$$


where $L=T+V$ is Lagrangian function, i.e., for the actual motion

$$
\delta \int_{t_{0}}^{t_{1}} L d t \leq 0 ; \quad \delta \mathbf{r}_{v}=0 \quad \text { with } \quad t=t_{0}, t_{1}
$$

Principle (7.1) was originally stated by Hamilton ${ }^{11}$ for the case of stationary holonomic systems with bilateral constraints under the action of potential forces, and was then extended by Ostrogradskii ${ }^{12}$ to the non-stationary case, and also to the case of non-potential forces. For holonomic systems with unilateral constraints, principles (7.1) and (7.3) were investigated by Stavrakova. ${ }^{13}$

\section{The principle of least action in Lagrangian form}

The first verbal formulation of the principle of least action was given by Maupertuis (1744) who then (1746) declared this principle as the universal law of motion and rest. ${ }^{14}$ : "General principle. When in nature a certain change occurs, the amount of action necessary for this change is the least possible. The amount of action is the product of the mass of the bodies by their velocity and the distance that they travel".

The mathematical formulation of the principle of least action for the case of a point mass was given by Euler. ${ }^{15}$ Lagrange $^{6}$ derived the principle for a system of point masses with bilateral constraints.

Below we will assume that the holonomic constraints imposed on the system are stationary, impacts possible in the system as it comes against unilateral constraints or leave them being absolutely elastic (coefficient of restitution $\delta=1$ ) Ref. 1 and the applied active forces are potential with a force function $U\left(\mathbf{r}_{1}, \ldots, \mathbf{r}_{N}\right)$ that is independent of time. Under these conditions the following energy integral exists

$$
H=T-U=h=\text { const }
$$

In fact, under these conditions, the actual displacements $d r_{\nu}=\dot{r}_{\nu} d t(\nu=1, \ldots, N)$ are among the virtual displacements. Multiplying Eq. (3.1) scalarly by $\mathrm{d} \mathbf{r}_{\nu}$, and summing over all $\nu$, we obtain, with the above assumptions, the equation

$$
\sum_{v} m_{v} \ddot{\mathbf{r}}_{v} \cdot d \mathbf{r}_{v}-d U=d(T-U)=0
$$

from which integral (8.1) follows. If the relations between the Cartesian and generalized coordinates are timeindependent, the Lagrangian function $L(q, \dot{q})=T+V$ will also be independent of time, and integral (8.1) will exist in this case also.

Left to itself, the mechanical system may choose its motions from motions with a given store of the total energy $h$, and therefore the set of motions being compared may be limited by condition (8.1). ${ }^{2}$

The actual motion will vary so that the initial position $A_{0}$ and the final position $A_{1}$ of the system remain unchanged. We will obtain the varied trajectory by producing at each instant of time $t$ the virtual displacement $\delta \mathbf{r}_{v}\left(\delta q_{i}\right)$ relative to the actual trajectory, where the instant of time $t+\delta t$ corresponds to the position $\mathbf{r}_{v}+\delta \mathbf{r}_{v}\left(q_{i}+\delta q_{i}\right)$ on a trajectory being compared. Since the total energy $H$ should be equal to $h$ on all the trajectories considered, from this the velocity at the position $\mathbf{r}_{v}+\delta \mathbf{r}_{v}$ is found. Thus, unlike earlier, the time is varied, and the duration of the motions being compared will differ from the duration of the actual motion between points $A_{0}$ and $A_{1}$. Below we will consider $\delta \mathbf{r}_{v}\left(\delta q_{i}\right)$ and $\delta t$ to be functions of time $t$ of class $C_{2} \cdot{ }^{16}$

We will integrate expression (3.5) of the d'Alembert-Lagrange principle with respect to $t$ in the range from $t_{0}$ to $t$ - the instant the final position $A_{1}$ is reached. We obtain

$$
0 \geq \int_{t_{0}}^{t_{1}}\left(\delta U-\sum_{v} m_{v} \ddot{\mathbf{r}}_{v} \cdot \delta \mathbf{r}_{v}\right) d t
$$

Since

$$
\int_{t_{0}}^{t_{1}} \ddot{\mathbf{r}}_{\mathrm{v}} \cdot \delta \mathbf{r}_{\mathrm{v}} d t=\left.\dot{\mathbf{r}}_{\mathrm{v}} \cdot \delta \mathbf{r}_{\mathrm{v}}\right|_{t_{0}} ^{t_{1}}-\int_{t_{0}}^{t_{1}} \dot{\mathbf{r}}_{\mathrm{v}} \cdot \delta \dot{\mathbf{r}}_{\mathrm{v}} d t ; \quad \delta \mathbf{r}_{\mathrm{v}}=0 \quad \text { with } \quad t=t_{0}, t_{1}
$$


the previous relation takes the form

$$
0 \geq \int_{t_{0}}^{t_{1}}(\delta U+\delta T) d t ; \quad \delta T=\sum_{v} m_{v} \dot{\mathbf{r}}_{\mathrm{v}} \cdot \delta \dot{\mathbf{r}}_{\mathrm{v}}
$$

Varying the energy integral (8.1), we obtain $\delta T=\delta U$, and consequently the last relation can be represented in the form

$$
\delta \int_{t_{0}}^{t_{1}} 2 T d t \leq 0 ; \quad \delta \mathbf{r}_{v}=0 \quad \text { with } \quad t=t_{0}, t_{1}
$$

It expresses the principle of least action in Lagrangian form for ideal smooth unilateral constraints.

The actual motion, being compared with other close motions occurring with the same constant energy $H$, satisfies condition (8.2); here, the variations of positions should be virtual displacements, and the initial and final positions of the system remain unvaried.

\section{The principle of least action in Jacobian form}

Jacobi ${ }^{17}$ iminated the time $t$ from the expression of action (8.2) using the energy integral and gave the principle of least action a geometric form.

Consider the configuration space defined by the line element ${ }^{2}$

$$
d s^{2}=\sum_{i j}^{n} a_{i j} d q_{i} d q_{j}
$$

and let us represent the kinetic energy of the system in the form

$$
T=\frac{1}{2} \sum_{i j}^{n} a_{i j} \dot{q}_{i} \dot{q}_{j}=\frac{1}{2} t^{\prime}\left(\frac{d s}{d \tau}\right)^{2}, \quad t^{\prime}=\frac{d t}{d \tau}
$$

where $\tau$ is a parameter. Let the family of surfaces $\tau\left(q_{1}, \ldots, q_{n}\right)=c$ intersect the actual trajectory of the system, and also infinitesimally close trajectories drawn through the points $A_{0}$ and $A_{1}$ of configuration space. Each of these curves can be considered to be specified by its coordinates represented as functions of $\tau$. Let the virtual displacements of the system from some point of the actual trajectory to a point of a being compared trajectory correspond to the same value of $\tau$, and let the positions $A_{0}$ and $A_{1}$ correspond to the values of $\tau_{0}$ and $\tau_{1}$ respectively.

We will express $\tau^{\prime}$ from Eq. (3.1) and replace $T$ with $U+h$; we then obtain

$$
t^{\prime}=\frac{1}{2 \sqrt{T}} \frac{d s}{d \tau}=\frac{1}{\sqrt{2(U+h)}} \frac{d s}{d \tau}
$$

and we represent relation (8.2) in the form

$$
\delta \int_{t_{0}}^{t_{1}} \sqrt{2(U+h)} \frac{d s}{d \tau} d \tau \leq 0, \quad \delta q_{i}=0 \quad \text { при } \quad \tau=\tau_{0}, \tau_{1}
$$

Thus, Lagrange variational principle with fixed lower and free upper bounds is transformed to Jacobi variational principle with fixed bounds $\tau_{0}$ and $\tau_{1}$ and fixed ends $A_{0}$ and $A_{1}$.

The Euler-Lagrange equations for problem (9.3) have the form ${ }^{2}$

$$
\frac{d}{d \tau}\left[\sqrt{2(U+h)} \frac{\partial}{\partial q_{s}^{\prime}} \sqrt{\sum_{i j} a_{i j} q_{i}^{\prime} q_{j}^{\prime}}\right]-\frac{\partial}{\partial q_{s}}\left[\sqrt{2(U+h)} \sqrt{\sum_{i j} a_{i j} q_{i}^{\prime} q_{j}^{\prime}}\right]=0, \quad s=1, \ldots, n
$$


$\left(q_{i}^{\prime}=d q_{i} / d \tau\right)$. These are the differential equations of the actual trajectory in the configuration space. The motion in time is found by integrating Eq. (9.2), which gives $t$ as a function of the parameter $\tau$.

If $d t \neq 0$ on the trajectory considered, then, as the parameter $\tau$, it is possible to select the time $t$ measured on the actual trajectory, for which integral (8.1) occurs. Here, the Eq. (9.4) take the form of Lagrange's equations of motion of the second kind

$$
\frac{d}{d t} \frac{\partial T}{\partial \dot{q}_{s}}-\frac{\partial T}{\partial q_{s}}=\frac{\partial U}{\partial q_{s}}, \quad s=1, \ldots, n
$$

which also proves the Jacobi principle.

Note $^{2}$ that in mechanical problems it is not possible to fix the values of $t_{0}, t_{1}, A_{0}, A_{1}$ and $h$ in advance.

\section{The Suslov-Voronets principle for non-holonomic systems}

Consider a mechanical system with the generalized coordinates $q_{\sigma}(\sigma=1, \ldots, r+b)$ which identically satisfy all holonomic constraints. Suppose, furthermore, that the system is subjected to $b$ non-holonomic constraints ${ }^{1}$

$$
\dot{q}_{r+\beta}-\sum_{\rho=1}^{r} u_{\beta \rho}\left(q_{\sigma}, t\right) \dot{q}_{\rho}-u_{\beta}\left(q_{\sigma}, t\right) \geq 0, \quad \beta=1, \ldots, b
$$

so that the virtual displacements satisfy the conditions

$$
\delta q_{r+\beta}-\sum_{\rho=1}^{r} u_{\beta \rho} \delta q_{\rho} \geq 0, \quad \beta=1, \ldots, b
$$

The operations of differentiation and variation are permutable only for $\rho=1, \ldots, r$, i.e.

$$
\frac{d}{d t} \delta q_{\rho}=\delta \dot{q}_{\rho}, \quad \rho=1, \ldots, r
$$

and for the remaining $b$ dependent velocities, from inequalities (10.1) and (10.2) we derive the relations

$$
\frac{d}{d t} \delta q_{r+\beta}-\delta \dot{q}_{r+\beta} \equiv \sum_{\rho=1}^{r}\left[\dot{u}_{\beta \rho} \delta q_{\rho}-\dot{q}_{\rho} \delta u_{\beta \rho}\right]-\delta u_{\beta}=\delta B_{\beta}, \quad \beta=1, \ldots, b
$$

The symbol $\delta B_{\beta}$ denotes expressions that are linear and homogeneous in $\delta q_{\beta}$. If Eq. (10.1) are integrable, then all $\delta B_{\beta} \equiv 0$.

We integrate the expression of the d'Alembert-Lagrange principle in generalized coordinates

$$
\delta U+\sum_{\sigma=1}^{r+b}\left(\frac{\partial T}{\partial q_{\sigma}}-\frac{d}{d t} \frac{\partial T}{\partial \dot{q}_{\sigma}}\right) \delta q_{\beta} \leq 0
$$

from $t_{0}$ to $t_{1}$

$$
\int_{t_{0}}^{t_{1}}\left[\delta U+\sum_{\sigma=1}^{r+b}\left(\frac{\partial T}{\partial q_{\sigma}}-\frac{d}{d t} \frac{\partial T}{\partial \dot{q}_{\sigma}}\right) \delta q_{\beta}\right] d t \leq 0
$$

assuming that, for $t=t_{0}$ and $t=t_{1}$, all independent variations $\delta q_{\rho}(\rho=1, \ldots, r)$ and consequently also dependent variations $\delta q_{r+b}$ are equal to zero $(\beta=1, \ldots, b)$.

We will have

$$
\int_{t_{0}}^{t_{1}} \frac{d}{d t} \frac{\partial T}{\partial \dot{q}_{\rho}} \delta q_{\rho} d t=\mid{ }^{t_{0}} \frac{\partial T}{t_{1}} \frac{\dot{q}_{\rho}}{{ }_{p}} \delta q_{\rho}-\int_{t_{0}}^{t_{1}} \frac{\partial T}{\partial \dot{q}_{\rho}} \frac{d}{d t} \delta q_{\rho} d t=-\int_{t_{0}}^{t_{1}} \frac{\partial T}{\partial \dot{q}_{\rho}} \delta \dot{q}_{\rho} d t, \quad \rho=1, \ldots, r
$$


and also, taking relations (10.3) into account

$$
\begin{aligned}
& \int_{t_{0}}^{t_{1}} \frac{d}{d T} \frac{\partial T}{\partial \dot{q}_{r+\beta}} \delta q_{r+\beta} d t=||_{t_{1}}^{t_{0}} \frac{\partial T}{\partial \dot{q}_{r+\beta}} \delta q_{r+\beta}-\int_{t_{0}}^{t_{1}} \frac{\partial T}{\partial \dot{q}_{r+\beta}} \frac{d}{d t} \delta q_{r+\beta} d t= \\
& =-\int_{t_{0}}^{t_{1}} \frac{\partial T}{\partial \dot{q}_{r+\beta}} \delta \dot{q}_{r+\beta} d t-\int_{t_{0}}^{t_{1}} \frac{\partial T}{\partial \dot{q}_{r+\beta}} \delta B_{\beta} d t, \quad \beta=1, \ldots, b
\end{aligned}
$$

Substituting these results into relation (10.4), we obtain the Suslov-Voronets principle ${ }^{1,18}$

$$
\int_{t_{0}}^{t_{1}}\left(\delta U+\delta T+\sum_{\beta=1}^{b} \frac{\partial T}{\partial \dot{q}_{r+\beta}} \delta B_{\beta}\right) d t \leq 0
$$

\section{Acknowledgement}

I wish to thank V. F. Zhuravlev for drawing my attention to the monograph Ref. 19.

\section{References}

1. Suslov GK. Theoretical Mechanics. Moscow and Leningrad: Gostekhizdat; 1946.

2. Chetayev NG. Theoretical Mechanics. Moscow: Nauka; 1987.

3. Lanczos C. The Variational Principles of Mechanics. Toronto: University Press; 1962.

4. Newton I. Mathematical Principles of Natural Philosophy (Philosophiae naturalis principia mathematica) (translated from the Latin by E. Mott), 1793; revised edition, edited by F. Cajori, Berkeley, CA: University of California Press; 1947.

5. Fourier JB. Mémoire sur la statique, contenant la démonstration du principe des vitesses virtuelles et la théorie des moments. École Polytechn J 1797-1798;2:20-60.

6. Lagrange G. Mécanique Analitique. Paris: Desaint; 1788.

7. Ostrogradskii MV. General reasoning in relation to moments of forces. Selected Papers. Leningrad: Izd Akad Nauk SSSR; 1958, $205-29$.

8. Gauss CF. Über ein neues allgemeines Grungesetz der Mechanik. J Reine Angew Math 1829:4; Werke; 1867, Vol. 5, $25-8$.

9. Chetayev NG. A modification of Gauss principle. Prikl Mat Mekh 1941;5(1):11-2.

10. Jourdain PE. Note on an analogue of Gauss principle of least constraint. Quart J Pure Appl Mathematics 1909;40:153-7.

11. Hamilton WR. On the application to dynamics of a general mathematical method previously applied to optics. Report of the 4th Meeting of the British Association for the Advancement of Science, Edinburgh, 1934. London; 1935: 513-8. Math Papers 1940;2:212-6.

12. Ostrogradsky M. Sur les integrales des équations générales de la dynamique. Bull Cl Phys-Math de l'Acad des Sc de St Pbg 1850;8(3):33-43.

13. Stavrakova NYe. The Hamilton-Ostrogradskii principle for a system with unilateral constraints. Prikl Mat Mekh 1965;29(4):738-41.

14. Maupertuis P-LM. Les lois de mouvement et du repos déduites d'un principe métaphysique. Mem Acad Roy Sci et Belles Lettres 1746:267-94.

15. Euler L. Methodus inveniendi lineas curvas maximi minimive proprietate gaudentes sive solutio problematis isopermetrici latissmo sensu accepti. Bousquet: Lausannae et Genevae; 1744.

16. Hölder O. Über die Prinzipien von Hamilton und Maupertuis. Nachricht Köm Ges Wissensch Göttingen Math-Phys Kl 1896;2:122-57.

17. Jacobi KG. Vorlesungen über Dynamik. Berlin: Reimer; 1884.

18. Voronets PV. On the equations of motion for non-holonomic systems. Mat Sb 1901;22(4):659-86.

19. Zhuravlev VF, Fufayev NA. Mechanics of Systems with Unilateral Constraints. Moscow: Nauka; 1993.

Translated by P.S.C. 